Ophthalmologe 2022 · 119:699-704 https://doi.org/10.1007/s00347-021-01552-9 Eingegangen: 17. Oktober 2021 Überarbeitet: 20. November 2021 Angenommen: 22. November 2021 Online publiziert: 22. Dezember 2021 (c) Der/die Autor(en) 2021

\title{
Effekt der „low-dose PDT“ auf die choriokapilläre Perfusion bei CCRCS
}

\section{Eine OCT-Angiographie-gestützte Studie}

\author{
E. Reifeltshammer (D) $\cdot$ N. Feucht $\cdot$ C. P. Lohmann $\cdot$ M. Maier \\ Klinik und Poliklinik für Augenheilkunde, Klinikum rechts der Isar der Technischen Universität München, \\ München, Deutschland
}

\section{Zusammenfassung}

Hintergrund: Bei Patienten mit chronischer Chorioretinopathia centralis serosa (cCRCS) soll die „low-dose photodynamische Therapie“ (PDT) über eine kurzfristige choriokapilläre Minderperfusion zu einem langfristigen vaskulären Umbau mit konsekutiver Reduktion der vaskulären Hyperpermeabilität und Leckage führen. Ob sich die verminderte Perfusion gänzlich normalisiert, bleibt jedoch ungeklärt. Hauptziel der retrospektiv angelegten Studie war es, das choriokapilläre Flusssignal nach „low-dose PDT" mittels optischer Kohärenztomographie-Angiographie (OCT-A) zu analysieren.

Patienten und Methoden: Eingeschlossen wurden 19 im Rahmen der "low-dose PDT" belichtete Areale an 16 Augen. Neben der Erhebung von Visus und Metamorphopsien wurden ein „enhanced depth imaging-OCT“ (EDI-OCT) und eine OCT-Angiographie mit Zentrierung auf das bei der "low-dose PDT" belichtete Areal angefertigt. Im Rahmen der vorliegenden Studie wurden das choriokapilläre Flusssignal sowie die chorioidale Dicke innerhalb eines genormten Bezirks sowohl im Bereich der PDT-Applikation als auch in einem unbeleuchteten Referenzareal in direkter Nähe mit gleicher Exzentrizität in Bezug auf die Fovea centralis retrospektiv ausgewertet.

Ergebnisse: Es konnten im Mittel eine Abnahme des choriokapillären Flusssignals um $33 \%(p<0,001)$ im belichteten Areal gegenüber dem Referenzareal sowie eine im Durchschnitt um $71 \mu \mathrm{m}(p=0,001)$ verminderte Aderhautdicke im Vergleich zum Referenzareal gezeigt werden; 7 von 10 Patienten beklagten trotz "low-dose PDT" langfristig Metamorphopsien, jedoch ergab sich durch die Therapie bei fast der Hälfte der Patienten eine Visusverbesserung.

Schlussfolgerungen: Durch die OCT-Angiographie konnte gezeigt werden, dass die „low-dose PDT“ im Bereich der Choriokapillaris ein vermindertes Blutflusssignal zurücklässt und somit das vaskuläre Remodelling die thrombosebedingte Hypoperfusion nicht gänzlich kompensiert.

\section{Schlüsselwörter}

Chorioretinopathia centralis serosa $\cdot$ Photodynamische Therapie · OCT-Angiographie ·

Choriokapillärer Blutfluss · Chorioidale Dicke

\section{Hintergrund und Fragestellung}

Die Chorioretinopathia centralis serosa (CRCS) ist eine Erkrankung des hinteren Augenpols, welche mit einer Verdickung der Aderhaut und einer serösen Abhebung der neurosensorischen Netzhaut einhergeht [5]. Männer sind bei einer Inzidenz von 9,9:100.000 etwa 6-mal häufiger betroffen als Frauen [11]. Das mittlere Erkrankungsalter liegt bei 45,2 Jahren [19].

Risikofaktoren sind hohe Kortisolspiegel, Autoimmunerkrankungen, eine $\mathrm{He}$ licobacter-pylori-Infektion, die Einnahme von Psychopharmaka, eine Typ-A-Persönlichkeitsstörung, Schlafstörungen, wie z. B. obstruktives Schlafapnoesyndrom, arteri- 

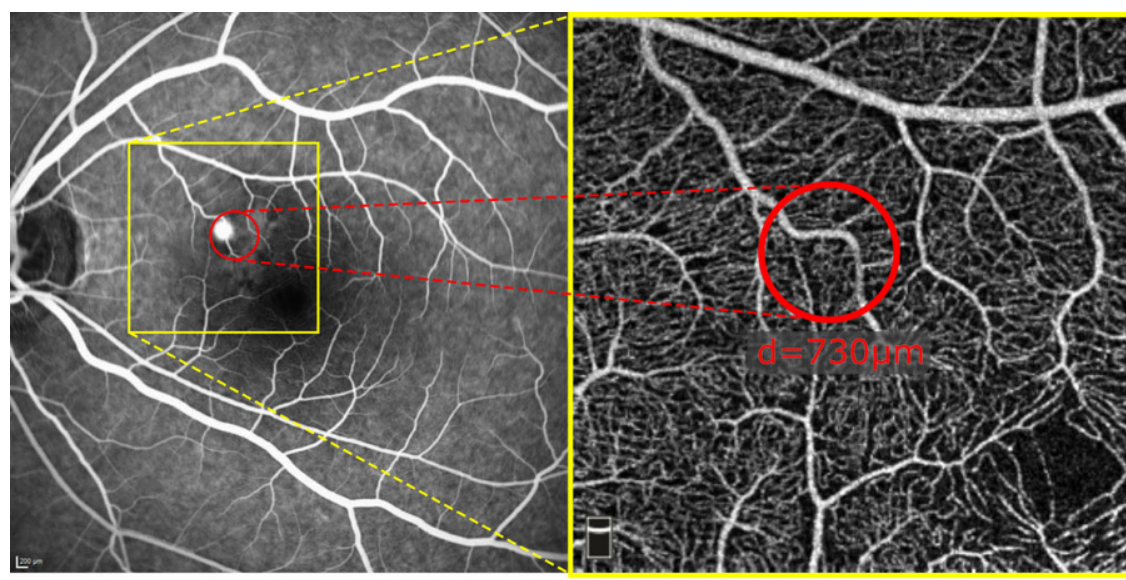

a

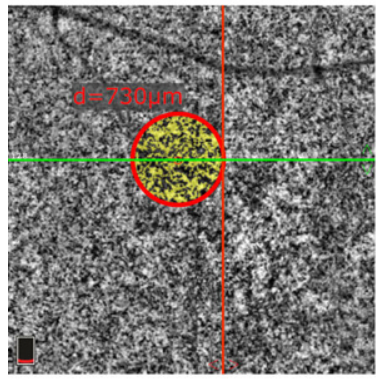

c

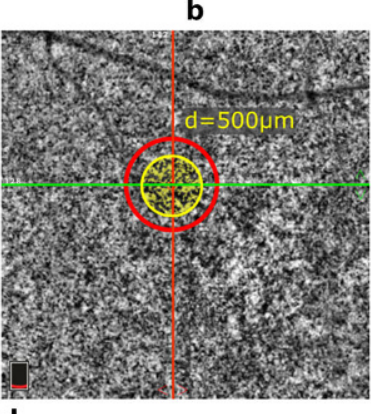

d

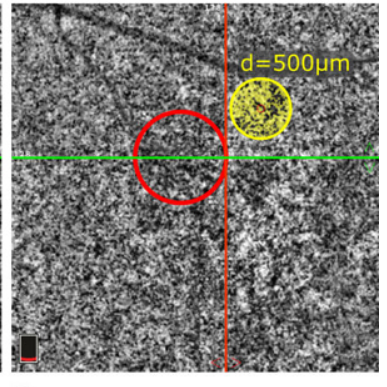

e

Abb. $1 \Delta$ Messung des Flusssignals. a Fluoreszenzangiographie, $30^{\circ}$ vor „low-dose PDT", mit Quellpunkt und belichtetem Bereich (roter Kreis, Durchmesser bei diesem Patienten $730 \mu \mathrm{m}$ ). b OCT-A des superfiziellen Kapillarplexus $(3 \times 3 \mathrm{~mm})$ nach PDT mit markiertem belichteten Bereich. c Darstellung desselben PDT-belichteten Bereichs in der choriokapillären Segmentierung. $\mathbf{d}$ Ausgewähltes Areal konstanter Größe (Durchmesser bei allen Patienten $500 \mu \mathrm{m}$ ) innerhalb des PDT-belichteten Bereichs, in welchem das Flusssignal gemessen wurde. e Ausgewähltes Areal konstanter Größe außerhalb des PDT-belichteten Bereichs (Referenzareal)

elle Hypertonie [12] sowie eine Schwangerschaft (besonders 3. Trimenon) [7].

Auch wenn die der CRCS zugrunde liegende Pathophysiologie noch nicht gänzlich geklärt ist, scheint eine verdickte Aderhaut (Pachychoroid, griech. pakhús = dick), welche auch am nicht erkrankten Partnerauge gefunden werden kann, eine zentrale Rolle einzunehmen $[8,15]$. In jüngster Zeit ergaben sich vielversprechende Erklärungsversuche auf molekularer Ebe$n e$, in deren Mittelpunkt eine Überaktivierung von Mineralokortikoidrezeptoren und die daraus resultierende chorioidale Hyperpermeabilität sowie Vasodilatation stehen [5].

Zur Einteilung der CRCS hat sich im klinischen Alltag die Unterscheidung zwischen einer akuten und einer chronischen Verlaufsform etabliert. Hierzu wurde eine arbiträre zeitliche Grenze bei 3 bis 6 Monaten gezogen, nach welcher eine Spontanheilung als unwahrscheinlich gilt und somit eine Therapie eingeleitet werden soll [5]. medikamentösen Therapie mit Mineralokortikoidantagonisten abgeraten $[1,13]$.

Typische Symptome einer akuten CRCS sind Verschwommensehen mit einem relativen Zentralskotom, Metamorphopsien, Dyschromatopsie, Mikropsie und eine verminderte Kontrastsensitivität, bei der chronischen Form hingegen steht eine reduzierte Sehschärfe im Vordergrund.

Im klinischen Alltag spielt die photodynamische Therapie in der Behandlung der chronischen CRCS eine zentrale Rolle.

Obgleich die Wirksamkeit der "full-dose PDT" $\left(6 \mathrm{mg} / \mathrm{m}^{2}\right.$ Verteporfin) vielfach belegt ist, ergaben die beiden folgenden Beobachtungen Anlass, den Einsatz der "fulldose PDT" zu überdenken. Cardillo Piccolino et al. konnten zeigen, dass es in $31 \%$ der von innen untersuchten Augen nach "full-dose PDT" zu sekundären RPE-Veränderungen kam, welche sie auf hypoxische Schäden durch die Okklusion der Choriokapillaris zurückführten [2].

Zudem gaben W.M. Chan et al. zu bedenken, dass der meist benigne Visusverlauf der chronischen CRCS nicht im Verhältnis zu einer möglichen exsudativen Makulopathie nach PDT steht, welche mit deutlich schlechterem Visus einhergeht [4].

Basierend auf dem Wissen einer dosisabhängigen Zytotoxizität [16], wurde eine Reduktion der Verteporfin-Dosis genauer untersucht. Chan et al. berichteten von mit "full-dose PDT“ vergleichbaren Erfolgen der "half-dose PDT" hinsichtlich Resorption der subretinalen Flüssigkeit bei deutlich geringerem Nebenwirkungsprofil [4].

Im Vergleich mit anderen lasergestützten Methoden konnte in der groß angelegten $(n=179)$, randomisierten, kontrollierten PLACE-Studie gezeigt werden, dass die "half-dose-PDT“ dem Mikropulslaser hinsichtlich anatomischer (Resorption der subretinalen Flüssigkeit), als auch funktioneller Endpunkte (Visus, retinale Sensitivität) überlegen ist. Als eine mögliche Begründung wird der Wirkmechanismus der PDT gesehen, welcher direkt auf die der CRCS zugrunde liegende Hyperpermeabilität der Choroidea abzielt, indem eine temporäre Okklusion der Choriokapillaris induziert wird $[16,18]$.

Obgleich bereits gezeigt werden konnte, dass sich innerhalb der vormals okkludierten Gefäße der Choriokapillaris neue 


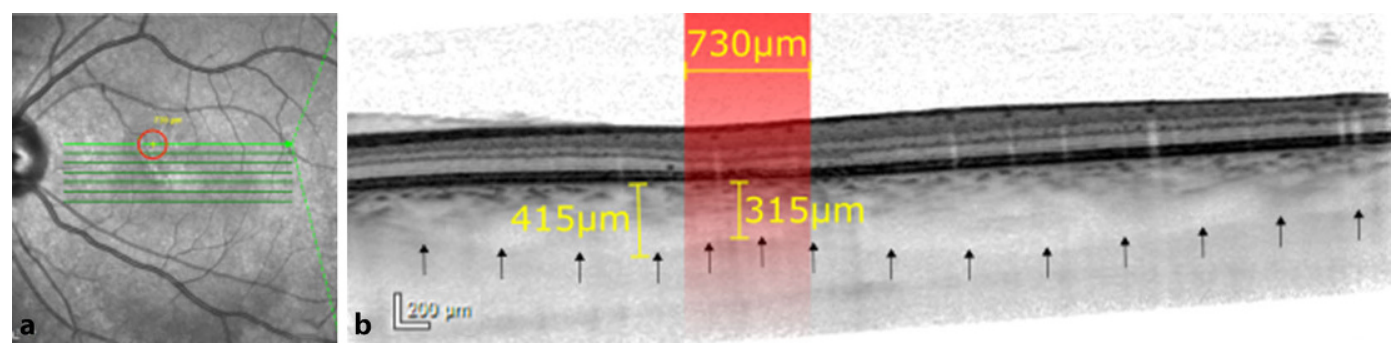

Abb. $2 \Delta$ Aderhautdickenmessung. a En-face-OCTmit markiertem PDT-belichtetem Bereich und Schnittebenen. b Scan mit rot markiertem belichteten Bereich, chorioskleralem Übergang (Pfeile) und Dickenmessungen innerhalb sowie außerhalb des PDT-belichteten Bereichs. Eine Verringerung der Aderhautdicke im belichteten Bereich ist bereits ohne Quantifizierung der Aderhautdicke im EDI-OCT deutlich zu erkennen

Lumina (,remodeling“) bilden [16], konnte bisher nicht geklärt werden, ob auch nach „low-dose PDT“ eine choriokapilläre Hypoperfusion persistiert und damit wie nach „full-dose PDT“ einen möglichen Risikofaktor für sekundäre choroidale Neovaskularisationen darstellt.

In dieser retrospektiven Studie soll der Einfluss einer Behandlung mit "low-dose PDT" sowohl auf den choriokapillären Blutfluss als auch auf die Dicke der Aderhaut mittels optischer KohärenztomographieAngiographie (OCT-A) untersucht werden. Dies soll Rückschlüsse auf die Frage erlauben, ob sich die durch die PDT-Behandlung induzierte choriokapilläre Hypoperfusion im Langzeitverlauf wieder vollständig normalisiert.

\section{Patienten und Methoden}

Das Patientenkollektiv bestand aus Patienten mit chronischer CRCS, welche am Klinikum rechts der Isar (München) zwischen April 2015 und Juni 2017 einer "lowdose PDT“ ("half-fluence PDT“, $6 \mathrm{mg} / \mathrm{m}^{2}$ Verteporfin, $25 \mathrm{~J} / \mathrm{cm}^{2}$ Lichtdosis entsprechend $42 \mathrm{~s}$ Belichtungszeit, Infusionsdauer $10 \mathrm{~min}$, Belichtung $15 \mathrm{~min}$ nach Infusionsstart, Lichtintensität $600 \mathrm{~mW} / \mathrm{cm}^{2}$, Zeiss VISULAS 690S PDT Laser, Carl Zeiss Meditec AG, Jena, Deutschland) unterzogen worden waren $(n=30)$. Da sowohl diffuse als auch fokale Leckageformen in die Studie eingeschlossen wurden, ergaben sich belichtete Areale mit Durchmessern zwischen 730 und $3500 \mu m$ (Durchschnitt $1700 \mu \mathrm{m}$ ). Die Behandlung erfolgte im Median 11 Monate nach Diagnosestellung durch Fluoreszenzangiographie (FLA). Ausschlusskriterien waren dabei die NichtPartizipation der Patienten $(n=14)$, der
Gebrauch systemischer Steroide sowie eine vorhergegangene Vorbehandlung durch PDT $(n=1)$.

In der daraus resultierenden Studienpopulation von 15 Patienten wurden Messungen des choriokapillären Blutflusses sowie der Aderhautdicke in 19 PDT-behandelten sowie 19 Referenzarealen in 16 Augen vorgenommen. Die aus diesen Messungen gewonnenen Daten wurden im Rahmen der vorliegenden Studie retrospektiv ausgewertet.

\section{Untersuchungsablauf}

Eine zur Planung der PDT-Behandlung erstellte, annotierte FLA wurde benützt, um das belichtete Areal wieder aufzusuchen und in einem $3 \times 3 \mathrm{~mm}$ großen OCTAngiographie-Fenster (Optovue RTVue XR Avanti, Optovue Inc., Freemont, CA, USA; Auflösung $5 \mu \mathrm{m}$ axial, $15 \mu \mathrm{m}$ lateral, $70.000 \mathrm{~A}-\mathrm{Scans}$ pro Sekunde) zu erfassen (- Abb. 1b). In diesem Fenster wurden zwei kreisförmige Gebiete (Durchmesser $=500 \mu \mathrm{m}$ ) bestimmt: eines innerhalb des PDT-belichteten Bereichs („,belichtetes Areal", - Abb. 1d), das andere außerhalb („Referenzareal“, - Abb. 1e). Die Wahl der Areale erfolgte unter Beachtung folgender Gesichtspunkte:

- möglichst gleiche Exzentrizität des belichteten und des Referenzareals in Bezug auf die Fovea centralis,

- Aussparung großer Gefäße des superfiziellen Gefäßplexus, um Projektionsartefakte gering zu halten,

- möglichst gleichmäßige OCT-A-Signalstärke,

- möglichst geringer Abstand zwischen belichtetem und Referenzareal.
In beiden Arealen wurde jene Fläche gemessen, in welcher ein Flusssignal detektiert werden konnte (Einheit $\mathrm{mm}^{2}$ ).

Darüber hinaus wurde mittels EDI-OCT (HRA + OCT Spectralis, Heidelberg Engineering $\mathrm{GmbH}$, Heidelberg, Deutschland) die Aderhautdicke (Einheit $\mu \mathrm{m}$ ) vom Unterrand der hyperreflektiven Linie, welche der basalen Begrenzung des RPE entspricht, bis zur hyporeflektiven Bande, die den chorioskleralen Übergang bildet, gemessen - in der gewählten Scanebene einerseits zentral im belichteten Areal sowie andererseits in möglichst geringem Abstand außerhalb desselben (• Abb. 2).

Die obigen Untersuchungen wurden einzeitig, im Median 7 Monate (min.: 3 Monate, max.: 31 Monate) nach "lowdose PDT"-Behandlung, durchgeführt. Folgende Befunde wurden sowohl vor als auch nach PDT-Behandlung erhoben: Fundusfoto, "spectral domain-OCT", Fundusautofluoreszenz sowie Visus und Metamorphopsien.

\section{Auswertung}

Um eine statistische Überrepräsentation von Patienten mit beidseitiger Erkrankung oder mehreren behandelten Arealen zu vermeiden, wurden die Daten zunächst areals- und dann patientenweise gemittelt.

Als Signifikanzniveau der statistischen Tests wurde ein $p$-Wert von $p=0,05$ festgelegt. Der choroidale Blutfluss sowie die Aderhautdicke im beleuchteten Areal wurden mittels t-Test für gepaarte Stichproben mit jenen des Referenzareals verglichen. Ebenso wurde der Visus prä-PDT jenem post-PDT mittels t-Test für gepaarte Stichproben gegenübergestellt. 

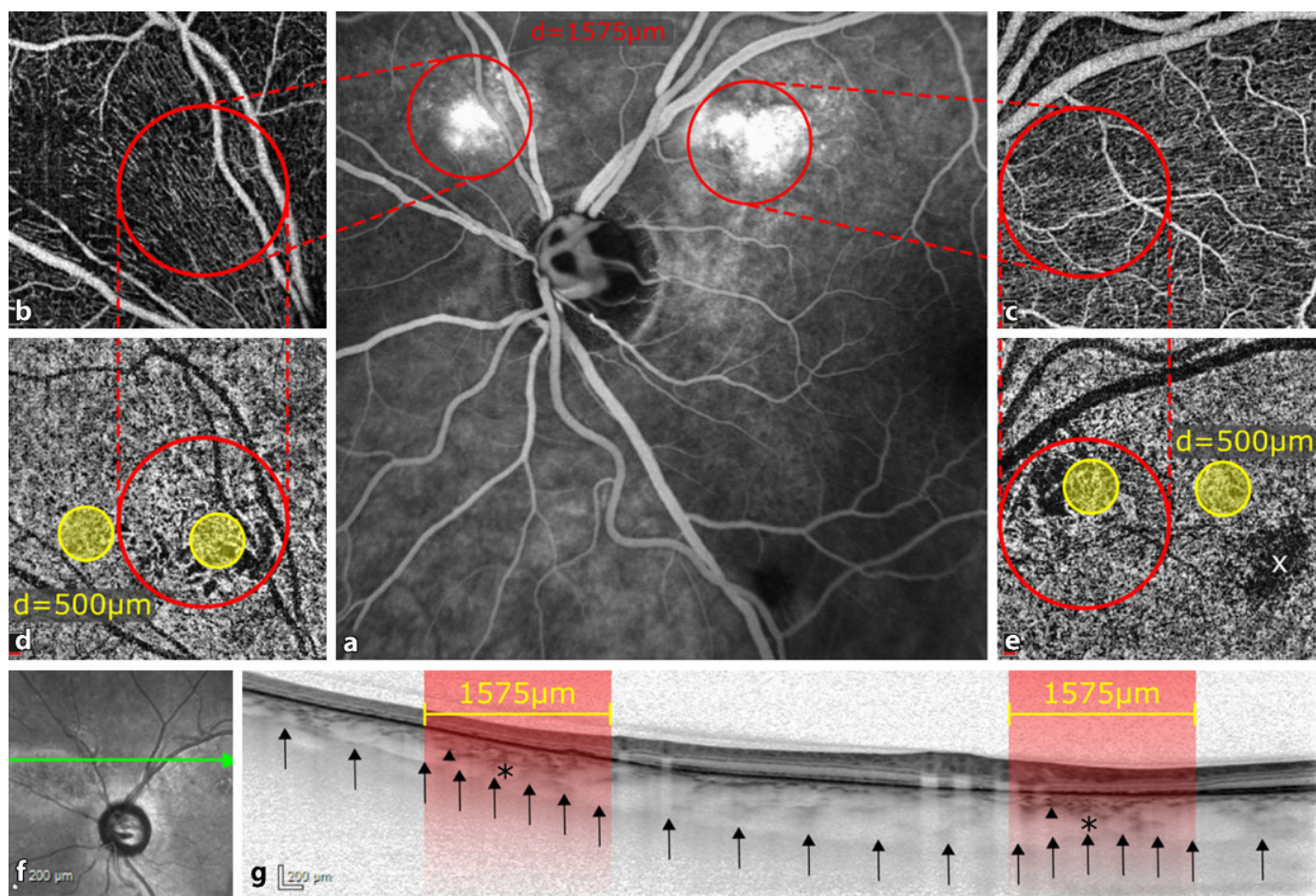

$1575 \mu \mathrm{m}$
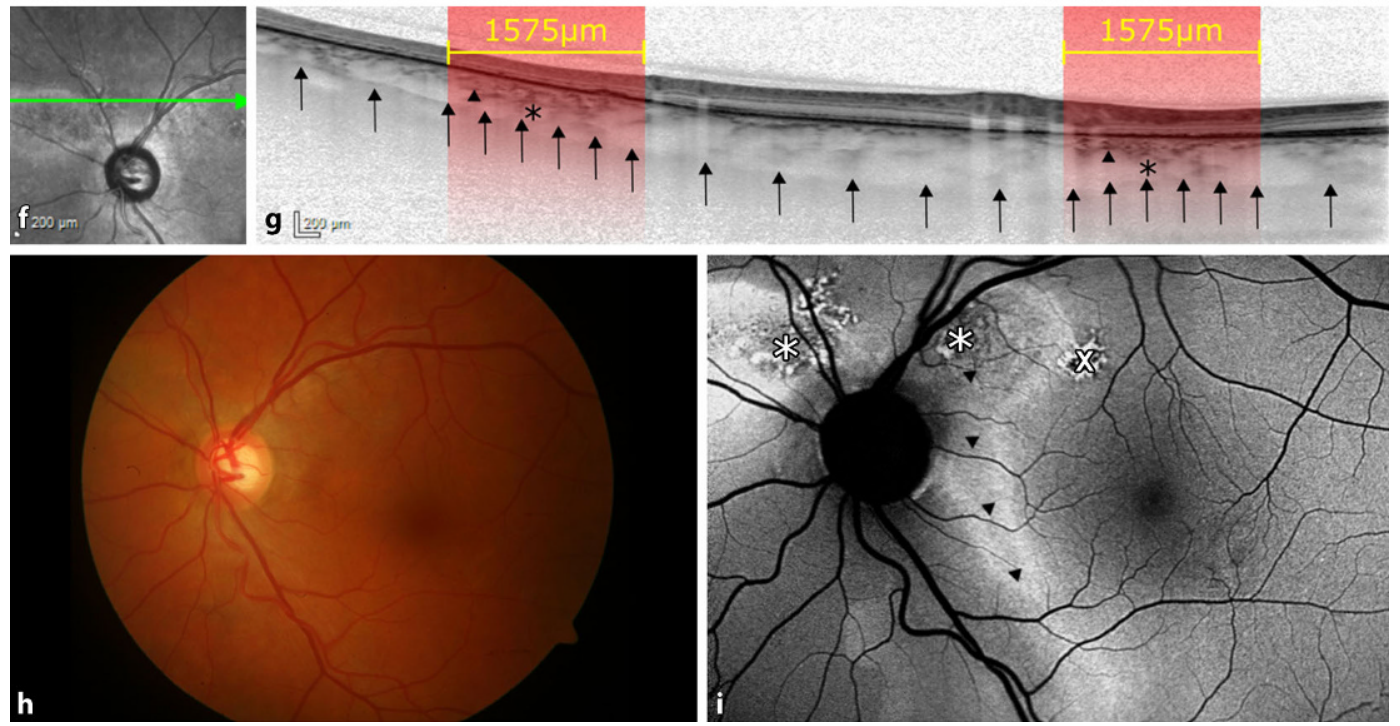

Abb. 3 ム Patientenbeispiel. a Fluoreszenzangiographie, $30^{\circ}$ vor "low-dose PDT", mit zwei Quellpunkten und belichteten Bereichen (rote Kreise, Durchmesser bei diesem Patienten $1575 \mu \mathrm{m}$ ). b, c OCT-A des superfiziellen Kapillarplexus $(3 \times 3 \mathrm{~mm}$ ) nach PDT mit markiertem belichteten Bereich. $d$, e Darstellung desselben PDT-belichteten Bereichs in der choriokapillären Segmentierung (rot), ausgewählte Areale konstanter Größe (Durchmesser bei allen Patienten $500 \mu \mathrm{m}$ ) innerhalb und außerhalb („Referenzareal“") des PDT-belichteten Bereichs, in welchen das Flusssignal gemessen wurde. Minderperfusion im Bereich einer alten Narbe (Kreuz). fEn-face-OCT mit Schnittebene. g B-Scan mit rot markiertem belichteten Bereich und chorioskleralem Übergang (Pfeile). Es zeigen sich eine erhöhte Transmission durch das retinale Pigmentepithel (Sternchen) sowie eine Verringerung der Dicke der Haller-Schicht. $\mathbf{h}$ Fundusfoto nach "low-dose PDT“. i Fundusautofluoreszenz. Vertikale hypofluoreszente Bahn („,descending tracts" nach Spaide und Klancnik [17]) mit hyperfluoreszentem Saum (Pfeilspitzen), RPE-Unruhe im Bereich der PDT-belichteten Areale (Sternchen), alte Narbe (Kreuz)

\section{Ergebnisse}

Die 15 Patienten $(12 \mathrm{~m}, 3 \mathrm{w})$ wiesen ein durchschnittliches Alter von 55,8 Jahren auf, bei allen war der Linsenstatus phak. Vor der "low-dose PDT“ wurden 12 Patienten mit Aldosteronantagonisten (Eplerenon bzw. Spironolacton) sowie 5 Patienten mit Carboanhydrasehemmern vorbehandelt. Bei insgesamt 2 Patienten wurde am Partnerauge eine CRCS diagnostiziert.

\section{Choriokapilläres Flusssignal in der} OCTA

Im beleuchteten Areal betrug das mittlere Flusssignal 0,09 $\pm 0,03 \mathrm{~mm}^{2}$, im Referenzareal wurden im Mittel $0,14 \pm 0,03 \mathrm{~mm}^{2}$ gemessen. Das im beleuchteten Areal bestimmte Flusssignal war im Mittel um $0,04 \mathrm{~mm}^{2}$ kleiner als im Referenzareal ( $p<0,001$; 95\%-Konfidenzintervall $(\mathrm{KI})=0,03 \mathrm{~mm}^{2}-0,06 \mathrm{~mm}^{2}$; t-Test für ge- paarte Stichproben). Die Abnahme des Mittelwerts um $33 \%$ stellt eine statistisch signifikante Veränderung dar.

\section{Choroidale Dicke}

Die mittlere chorioidale Dicke betrug im beleuchteten Areal 299,2 $\pm 116,0 \mu \mathrm{m}$, im Referenzareal 370,1 $\pm 135,0 \mu \mathrm{m}$. Im beleuchteten Areal war die Aderhaut im Mittel um 70,9 $\mathrm{mm}$ dünner als im Referenz- 
areal ( $p=0,001 ; 95 \%-\mathrm{KI}=34,0-108,0 \mu \mathrm{m}$; $\mathrm{t}$-Test für gepaarte Stichproben). Die Abnahme des Mittelwerts um 19\% ergibt eine statistisch signifikante Änderung.

Aufgrund mangelnder Aufnahmequalität konnte die Messung einer Patientin nicht in die statistische Auswertung einbezogen werden.

\section{Visus und Metamorphopsien}

Der Visus am beleuchteten Auge hat sich nach PDT-Behandlung bei 7 Patienten (47\%) verbessert, bei 5 Patienten (33\%) nicht verändert und bei 3 Patienten (20\%) verschlechtert. Im Durchschnitt verbesserte sich der Visus im behandelten Auge um $0,12 \log M A R(p=0,12 ; \mathrm{t}$-Test für gepaarte Stichproben). Bei 3 von 10 Patienten mit Metamorphopsien verschwanden diese nach der PDT-Behandlung.

\section{Patientenbeispiel}

Das folgende Beispiel (• Abb. 3) zeigt einen 49-jährigen Patienten mit chronischer CRCS, der in der FLA Leckagen in zwei peripher gelegenen Arealen aufwies (- Abb. 3a), welche in Folge mittels "lowdose PDT" behandelt wurden. Im Langzeitverlauf konnten mithilfe der OCT-A in den ehemals belichteten Arealen persistierende Hypoperfusionen auf Ebene der Choriokapillaris nachgewiesen werden (s. Areale in - Abb. 3d, e).

\section{Diskussion}

In dieser Studie konnte eine statistisch signifikante Differenz $(p<0,001)$ des choriokapillären Flusssignals zwischen dem PDTbelichteten Areal und dem unbelichteten Referenzareal gezeigt werden. Somit ist davon auszugehen, dass die "low-dose PDT" eine persistierende Minderperfusion auf Ebene der Choriokapillaris hinterlässt und damit wie die "full-dose PDT" einen möglichen Risikofaktor für sekundäre choroidale Neovaskularisationen darstellt.

Schlötzer-Schrehardt et al. konnten bereits eine Woche nach "full-dose PDT" in zwei von drei Fällen elektronenmikroskopisch Rekanalisationen im Bereich der vormals okkludierten Choriokapillaris im Sinne eines vaskulären Remodellings nachweisen [16]. Da in der vorliegenden Studie der choriokapilläre Fluss im Median 7 Monate nach „low-dose PDT" gemessen wurde, wird ein noch nicht abgeschlossenes vaskuläres Remodelling als unwahrscheinlich erachtet.

Die choriokapilläre Perfusion nach „low-dose PDT“ wurde mittels OCT-Angiographie bereits in mehreren Studien untersucht, es konnte jedoch noch keine signifikante Veränderung beobachtet werden. Xu et al. untersuchten die Ebenmäßigkeit des Flussbildes vor und nach PDTBehandlung rein qualitativ und gingen von einer Normalisierung der Perfusion in $97 \%$ der Fälle aus [20]. Dabei räumen die Autoren selbst ein, dass quantitative Flussanalysen sowie ein längerer Beobachtungszeitraum nötig sein könnten, um genauere Aussagen zu treffen. S.Y. Chan et al. liefern in ihrer Arbeit quantitative Messwerte [3], jedoch wird das Flusssignal über einen $3 \times 3 \mathrm{~mm}$-Bereich gemittelt und in Relation zum Partnerauge gesetzt. Möglicherweise erlaubt erst die Wahl kleinerer, vollständig in- bzw. außerhalb des belichteten Areals gelegener Messbereiche den Nachweis signifikanter Perfusionsdefizite.

Ebenso konnte in dieser Studie eine statistisch signifikante Abnahme $(p=0,001)$ der Aderhautdicke zwischen dem PDT-belichteten Areal und dem unbelichteten Referenzareal gezeigt werden.

Die gemessenen Dicken können jedoch nicht direkt mit Messungen der subfovealen Dicke anderer Studien verglichen werden, da in allen Fällen das durch die „lowdose PDT" beleuchtete Areal extrafoveal lag. Dennoch konnten sowohl Izumi et al. als auch Maruko et al. eine Verringerung der Aderhautdicke im Zuge einer "lowdose PDT" bei CRCS nachweisen [9, 14].

Jirarattanasopa et al. beobachteten eine erhöhte choroidale Dicke bei verschiedenen Formen der CRCS im Bereich der Leckagen in der FLA sowie in Arealen choroidaler Hyperpermeabilität in der Indocyaningrün-Angiographie [10]. Im Umkehrschluss könnte eine Abnahme der choroidalen Dicke nach „low-dose PDT“ auf eine verminderte vaskuläre Hyperpermeabilität schließen lassen, was Maruko et al. bereits andeuteten [14].

Nachteile der vorliegenden Studie ergeben sich einerseits durch die Einzeitigkeit der OCT-A-Aufnahmen, welche keine Ver- gleiche ein und desselben Areals prä und post PDT-Behandlung erlauben, sowie andererseits durch die Varianz, welche durch die Wahl der Messareale bedingt ist.

\section{Fazit für die Praxis}

In der vorliegenden Studie konnte nach „lowdose PDT" eine persistierende Hypoperfusion auf Ebene der Choriokapillaris nachgewiesen werden.

Dennoch gilt die "half-dose PDT“ nach wie vor als valide Therapieoption der chronischen CRCS. Die wiederholte Belichtung desselben Netzhautareals ist jedoch aufgrund der kumulativen Toxizität kritisch zu sehen.

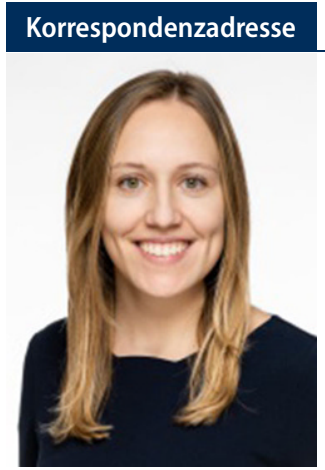

\section{E. Reifeltshammer}

Klinik und Poliklinik für Augenheilkunde, Klinikum rechts der Isar der Technischen Universität München Ismaninger Str. 22, 81675 München, Deutschland

reifeltshammer.elisa@gmail.com

Funding. Open Access funding enabled and organized by Projekt DEAL.

\section{Einhaltung ethischer Richtlinien}

Interessenkonflikt. E. Reifeltshammer, N. Feucht, C.P. Lohmann und M. Maier geben an, dass kein Interessenkonflikt besteht.

Für diesen Beitrag wurden von den Autoren keine Studien an Menschen oder Tieren durchgeführt. Für die aufgeführten Studien gelten die jeweils dort angegebenen ethischen Richtlinien.

Open Access. Dieser Artikel wird unter der Creative Commons Namensnennung 4.0 International Lizenz veröffentlicht, welche die Nutzung, Vervielfältigung, Bearbeitung, Verbreitung und Wiedergabe in jeglichem Medium und Format erlaubt, sofern Sie den/die ursprünglichen Autor(en) und die Quelle ordnungsgemäß nennen, einen Link zur Creative Commons Lizenz beifügen und angeben, ob Änderungen vorgenommen wurden. 
Die in diesem Artikel enthaltenen Bilder und sonstiges Drittmaterial unterliegen ebenfalls der genannten Creative Commons Lizenz, sofern sich aus der Abbildungslegende nichts anderes ergibt. Sofern das betreffende Material nicht unter der genannten Creative Commons Lizenz steht und die betreffende Handlung nicht nach gesetzlichen Vorschriften erlaubt ist, ist für die oben aufgeführten Weiterverwendungen des $\mathrm{Ma}$ terials die Einwilligung des jeweiligen Rechteinhabers einzuholen.

Weitere Details zur Lizenz entnehmen Sie bitte der Lizenzinformation auf http://creativecommons.org/ licenses/by/4.0/deed.de.

\section{Literatur}

1. Berufsverbandes der Augenärzte Deutschlands, RetinologischeGesellschaft (2020)Stellungnahme Chorioretinopathia centralis serosa (CCS)

2. Cardillo Piccolino F, Eandi CM, Ventre L et al (2003) Photodynamic therapy for chronic central serous chorioretinopathy. Retina 23:752-763

3. Chan SY, Pan CT, Wang Q et al (2019) Optical coherent tomographic angiographic pattern of the deep choroidal layer and choriocapillaris after photodynamic therapy for central serous chorioretinopathy. Graefes Arch Clin Exp Ophthalmol 257:1365-1372

4. Chan WM, Lai TY, Lai RY et al (2008) Safety enhanced photodynamic therapy for chronic central serous chorioretinopathy: one-year results of a prospective study. Retina 28:85-93

5. Daruich A, Matet A, Dirani A et al (2015) Central serous chorioretinopathy: recent findings and new physiopathology hypothesis. Prog Retin Eye Res 48:82-118

6. Daruich A, Matet A, Marchionno L et al (2017) Acute central serous chorioretinopathy: factors influencing episode duration. Retina 37:1905-1915

7. Haimovici R, Koh S, Gagnon DR et al (2004) Risk factors for central serous chorioretinopathy: acasecontrol study. Ophthalmology 111:244-249

8. Imamura Y, Fujiwara T, Margolis R et al (2009) Enhanced depth imaging optical coherence tomography of the choroid in central serous chorioretinopathy. Retina 29:1469-1473

9. Izumi T, Koizumi H, Maruko I et al (2017) Structural analyses of choroid after half-dose verteporfin photodynamic therapy for central serous chorioretinopathy. $\mathrm{Br} J$ Ophthalmol 101:433-437

10. Jirarattanasopa P, Ooto S, Tsujikawa A et al (2012) Assessment of macular choroidal thickness by optical coherence tomography and angiographic changes in central serous chorioretinopathy. Ophthalmology 119:1666-1678

11. Kitzmann AS, Pulido JS, Diehl NN et al (2008) The incidence of central serous chorioretinopathy in Olmsted County, Minnesota, 1980-2002. Ophthalmology 115:169-173

12. Liu B, Deng T, Zhang J (2016) Risk factors for central serous chorioretinopathy: a systematic review and meta-analysis. Retina 36:9-19

13. Lotery A, Sivaprasad S, O'connell A et al (2020) Eplerenone for chronic central serous chorioretinopathy in patients with active, previously untreated disease for more than 4 months $(\mathrm{VICl})$ : a randomised, double-blind, placebo-controlled trial. Lancet 395:294-303

14. Maruko I, lida T, Sugano $Y$ et al (2010) Subfoveal choroidal thickness after treatment of
Effect of low-dose PDT on perfusion of the choriocapillaris in cCSCR. An OCT-angiography-based study

Background: In patients with chronic central serous chorioretinopathy (CCSCR) it is assumed that low-dose photodynamic therapy (PDT) induces short-term hypoperfusion of the choriocapillaris, which leads to long-term choroidal vascular remodeling and subsequent reduction of vascular hyperpermeability and leakage; however, it remains unclear whether the hypoperfusion completely returns to normal.

The main aim of the retrospective study was to analyze the choroidal flow signal after treatment with low-dose PDT by optical coherence tomography-angiography (OCT-A). Patients and methods: In this study 16 eyes with a total of 19 treated areas were included. Aside from visual acuity and metamorphopsia checks, all patients underwent enhanced depth imaging-OCT (EDI-OCT) and OCT-A in the areas treated with lowdose PDT. In the present study, the flow signal in the choriocapillaris and choroidal thickness were measured retrospectively both in the PDT-treated area and in a nontreated reference area located in close proximity with similar eccentricity to the center of the fovea.

Results: The study found a mean reduction of $33 \%(p<0.001)$ of the choroidal flow signal in the treated area compared to the reference area and an average decrease in choroidal thickness of $71 \mu \mathrm{m}(p=0.001)$. In the long term 7 out of 10 patients suffered from metamorphopsia despite the low-dose PDT; however, visual acuity was improved in almost half of the patients by the treatment.

Conclusion: Using OCT-A it could be shown that at the choriocapillaris level, hypoperfusion persists in spite of low-dose PDT. Thus, the choroidal vascular remodeling is not able to completely compensate for the hypoperfusion induced by thrombosis.

\section{Keywords}

Central serous chorioretinopathy · Photodynamic therapy · Optical coherence tomography angiography · Choriocapillary blood flow . Choroidal thickness

central serous chorioretinopathy. Ophthalmology 117:1792-1799

15. Mrejen S, Spaide RF (2013) Optical coherence tomography: imaging of the choroid and beyond. Surv Ophthalmol 58:387-429

16. Schlotzer-Schrehardt U, Viestenz A, Naumann GO et al (2002) Dose-related structural effects of photodynamic therapy on choroidal and retinal structures of human eyes. Graefes Arch Clin Exp Ophthalmol 240:748-757

17. Spaide RF, Klancnik JM Jr. (2005) Fundus autofluorescence and central serous chorioretinopathy. Ophthalmology 112:825-833

18. van Dijk EHC, Fauser S, Breukink MB et al (2018) Half-dose photodynamic therapy versus highdensity subthreshold micropulse laser treatment in patients with chronic central serous chorioretinopathy: the PLACE trial. Ophthalmology 125:1547-1555

19. Wang M, Sander B, la Cour M et al (2005) Clinical characteristics of subretinal deposits in central serous chorioretinopathy. Acta Ophthalmol Scand 83:691-696

20. Xu Y, Su Y, Li L et al (2017) Effect of photodynamic therapy on optical coherence tomography angiography in eyes with chronic central serous chorioretinopathy. Ophthalmologica 237:167-172 